\title{
Comparative transcriptome analyses of genes involved in sulforaphane metabolism at different treatment in Chinese kale using full-length transcriptome sequencing
}

Qiuyun Wu, Junwei Wang, Shuxiang Mao, Haoran Xu, Qi Wu, Mantian Liang, Yiming Yuan, Mingyue Liu and Ke Huang ${ }^{*}$ (D)

\begin{abstract}
Background: Sulforaphane is a natural isothiocyanate available from cruciferous vegetables with multiple characteristics including antioxidant, antitumor and anti-inflammatory effect. Single-molecule real-time (SMRT) sequencing has been used for long-read de novo assembly of plant genome. Here, we investigated the molecular mechanism related to glucosinolates biosynthesis in Chinese kale using combined NGS and SMRT sequencing.

Results: SMRT sequencing produced 185,134 unigenes, higher than 129,325 in next-generation sequencing (NGS). $\mathrm{NaCl}(75 \mathrm{mM})$, methyl jasmonate (MeJA, $40 \mu \mathrm{M})$, selenate (Se, sodium selenite $100 \mu \mathrm{M})$, and brassinolide (BR, $1.5 \mu \mathrm{M})$ treatment induced 6893, 13,287, 13,659 and 11,041 differentially expressed genes (DEGs) in Chinese kale seedlings comparing with control. These genes were associated with pathways of glucosinolates biosynthesis, including phenylalanine, tyrosine and tryptophan biosynthesis, cysteine and methionine metabolism, and glucosinolate biosynthesis. We found $\mathrm{NaCl}$ decreased sulforaphane and glucosinolates (indolic and aliphatic) contents and downregulated expression of cytochrome P45083b1 (CYP83b1), S-alkyl-thiohydroximatelyase or carbon-sulfur lyase (SUR1) and UDP-glycosyltransferase 74B1 (UGT74b1). MeJA increased sulforaphane and glucosinolates contents and upregulated the expression of CYP83b1, SUR1 and UGT74b1; Se increased sulforaphane; BR increased expression of CYP83b1, SUR1 and UGT74b1, and increased glucosinolates contents. The desulfoglucosinolate sulfotransferases ST5a_b_c were decreased by all treatments.

Conclusions: We confirmed that $\mathrm{NaCl}$ inhibited the biosynthesis of both indolic and aliphatic glucosinolates, while MeJA and BR increased them. MeJA and BR treatments, conferred the biosynthesis of glucosinolates, and Se and MeJA contributed to sulforaphane in Chinese kale via regulating the expression of CYP83b1, SUR1 and UGT74b1.
\end{abstract}

Keywords: Single-molecule real-time sequencing, Next-generation sequencing, Chinese kale, Sulforaphane, De novo assembly

\footnotetext{
* Correspondence: huangkeqy@hotmail.com

College of Horticulture and Landscape, Hunan Agricultural University, No.1

Nongda Road, Furong District, Changsha 410128, Hunan Province, China
}

(c) The Author(s). 2019 Open Access This article is distributed under the terms of the Creative Commons Attribution 4.0 International License (http://creativecommons.org/licenses/by/4.0/), which permits unrestricted use, distribution, and reproduction in any medium, provided you give appropriate credit to the original author(s) and the source, provide a link to the Creative Commons license, and indicate if changes were made. The Creative Commons Public Domain Dedication waiver (http://creativecommons.org/publicdomain/zero/1.0/) applies to the data made available in this article, unless otherwise stated. 


\section{Highlights}

1. $\mathrm{NaCl}$ reduced sulforaphane and glucosinolate contents in Chinese kale.

2. MeJA, BR, and Se increased sulforaphane and/or glucosinolate contents.

3. SMRT sequencing generated 185,134 unigenes, higher than 129,325 in NGS.

4. Combined NGS/SMRT sequencing identified DEGs, CYP83b1, SUR1, and UGT74b1.

\section{Background}

Sulforaphane is a natural isothiocyanate available from widely consumed cruciferous vegetables, including broccoli, radish, and kale. Multiple characteristics of sulforaphane have been widely reported during the past two decades. Sulforaphane has various effects including antioxidant, antitumor formation and anti-inflammatory effect [1-5]. This compound has been shown to suppress or prevent tumor formation and development $[2,5]$.

One reason of for the great interest on sulforaphane is that it is an inducer of phase 2 enzymes, including phase 2 detoxification enzymes glucuronyltransferase (GT) and $\mathrm{NAD}(\mathrm{P}) \mathrm{H}$ :quinone reductase 1 (NQO1) $[2,6,7]$. Moreover, sulforaphane could stimulate the accumulation of glutathione, which plays important roles in cells including antioxidation, immune response, detoxification, and cancer pathogenesis [8]. For instance, sulforaphane has been reported to induce apoptosis and cell cycle arrest of human colon cancer cells by increasing cyclins A and B1 expression [2]; the administration of sulforaphane shows antitumor formation [9]; sulforaphane inhibits the $A \beta 1-$ 42-induced excessive secretion of interleukin-1 $\beta$ (IL-1 $\beta$ ) in human microglia-like cells via activation of nuclear factor erythroid 2-related factor 2 (Nrf2), a bZIP transcription factor and an inducer of phase 2 genes [10].

Sulforaphane, 1-isothiocyanato-4-(methyl-sulfinyl) butane, is a hydrolysis product of glucosinolate, 4-(methylsulfinyl)butyl glucosinolate, to be exact $[11,12]$. Glucosinolate and its hydrolysis products, including isothiocyanates, are the secondary metabolites in plants, especially in various kinds of edible cruciferous vegetables [11]. The formation of glucosinolate is an enzymatic reaction, including cytochrome P450 monooxygenases, methylthioalkylmalate synthases (MAMs),S-alkyl-thiohydroximatelyase or carbon-sulfur lyase (SUR1) $[13,14]$. The biosynthesis and accumulation of glucosinolates as well as sulforaphane is influenced by temperature, processing methods and abiotic stresses [11, 15-18]. Esfandiari et al. showed that $\mathrm{NaCl}$ treatment $(80$ and $160 \mathrm{mM})$ decreased the contents of glucosinolates (including gluconapin, 4-methoxyglucobrassicin and neoglucobrassicin) and increased sulforaphane, and the administration of salicylic acid (SA, $100 \mu \mathrm{M})$ lowered 80 $\mathrm{mM} \mathrm{NaCl}$-reduced gluconapin in Brassica oleracea [17].
They confirmed that $\mathrm{NaCl}$ and SA co-treatment induced sulforaphane and isothiocyanates accumulation was independent of the biosynthetic genes of cytochrome P45083b1 (CYP83b1), CYP83a1 and CYP79b2, but associated with the increased hydrolytic enzymes of myrosinase (MYO) and epithiospecifier moifier 1 (ESM1) [17]. Other researches showed the indole-3-acetic acid, methyl jasmonate (MeJA) and brassinosteroids treatments upregulated the production of glucosinolates $[19,20]$,whereas the contents of glucosinolates could be decreased under selenium (Se) stress [21, 22]. Tian et al. showed the expression of CYP79b2, CYP83b1, and CYP83a1 were significantly downregulated in B. oleracea following Se treatment [22]. $\mathrm{Ku}$ et al. performed a comparative transcriptome analysis in two broccoli cultivars under MeJA treatment and identified that the glucosinolates' core structure biosynthesis genes (including CYP79b2, UDP-glycosyltransferase 74B1, UGT74b1, SUR1) were upregulated by MeJA [23]. Several comparative transcriptome analyses in Chinese kale $(B$. oleracea var. alboglabra Bailey) [24, 25] and watercress [26] showed that the expression patterns of aforementioned genes related to glucosinolates biosynthesis were changed in different tissues. These reports demonstrate the complex and diversity glucosinolates biosynthesis and metabolism by different stresses and in different plants. It has been reported that the influence of stress-induced biosynthesis of sulforaphane is dependent on plant variety and growth conditions $[17,27]$. However, the causality of abiotic stresses-induced dysregulation of sulforaphane and glucosinolates biosynthesis has yet to be clearly explored, and the underlying mechanisms should be dissected.

In comparison with the next (second)-generation sequencing (NGS), the third-generation transcriptome (single-molecule real-time, SMRT) sequencing produced more complete transcriptome data with longer reads. SMRT sequencing has been widely used for plant genome assembly [28]. The problem of higher error rate in SMRT sequencing data could be resulted by correcting with NGS-generated short reads. Here we investigated the molecular mechanisms related to glucosinolates biosynthesis under different abiotic stresses in Chinese kale using combined NGS and SMRT sequencing data. Chine kale is a native Chinese brassica vegetable with good flavor and high content of vitamin $\mathrm{C}$, total phenolics, and glucosinolates. The market demand of Chinese kale sprouts is increasing due to the rich in glucosinolates. Transcriptome with complete and full-length reads would be to dissect molecular mechanisms of glucosinolates biosynthesis in Chinese kale.

\section{Results}

Sulforaphane and glucosinolates contents were changed by different treatments

We firstly treated with Chinese kale seedlings with series of $\mathrm{NaCl}, \mathrm{Se}, \mathrm{MeJA}$ and $\mathrm{BR}$ to select the optimal 
concentrations. We confirmed all treatments induced dose-dependent increment or reduction in sulforaphane contents (Fig. 1a). Treatment with $\mathrm{NaCl}(0-150 \mathrm{mM})$ and BR $(0-3.0 \mu \mathrm{M})$ significantly reduced sulforaphane. Lower concentrations of MeJA $(0-40 \mu \mathrm{M})$ and Se $(0-$ $100 \mu \mathrm{M})$ increased sulforaphane, while overdose MeJA $(>40 \mu \mathrm{M})$ and $\mathrm{Se}(>100 \mu \mathrm{M})$ decreased sulforaphane. We chose $75 \mathrm{mM} \mathrm{NaCl}, 1.5 \mu \mathrm{M} \mathrm{BR}, 40 \mu \mathrm{M}$ MeJA and $100 \mu \mathrm{M}$ Se as optimal concentrations for the further treatments.
We confirmed that the contents of sulforaphane (Fig. 1b) and glucosinolates (Fig. 1c, total, aliphatic and indolic) in Chinese kale seedlings were significantly reduced by $\mathrm{NaCl}$ treatment. Se and MeJA treatments obviously increased the contents of sulforaphane in kale seedlings in comparison with control ( $p<0.01$; Fig. 3a), and MeJA and BR treatments significantly increased the contents of total and aliphatic glucosinolates $(p<0.01$, Fig. 1c). The content of aromatic glucosinolates was only increased by Se treatment $(p<0.01)$ compared with

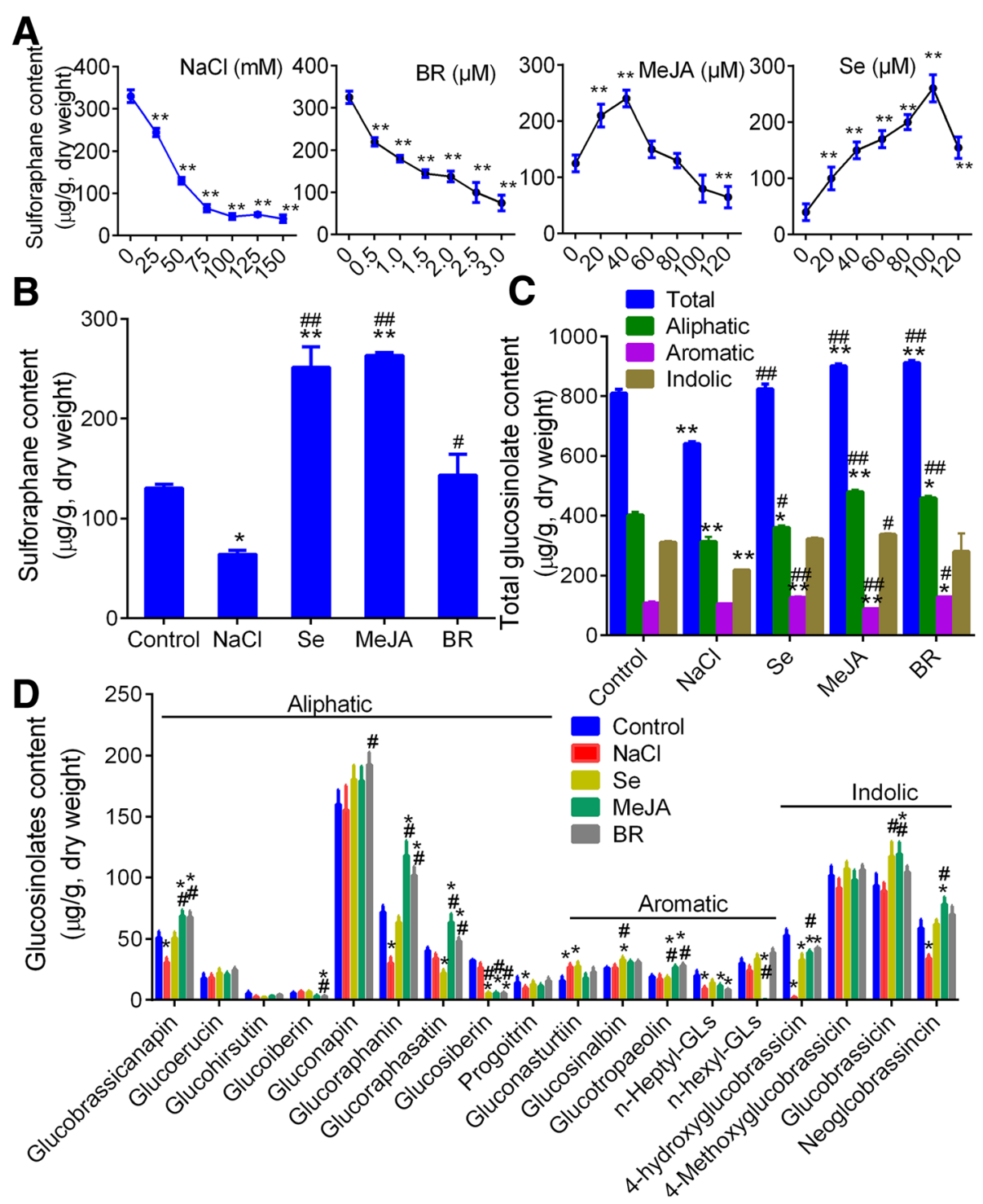

Fig. 1 The content of sulforaphane and glucosinolates in Chinese kale seedlings in response to different treatments. a the content of sulforaphane in Chinese kale seedlings under different treatments with series concentrations. $\mathbf{b}$ the content of sulforaphane under different treatments with optimal contents. $\mathbf{c}$ the total content of 18, aliphatic, aromatic and indolic glucosinolates in Chinese kale seedlings (dry weight, g). $\mathbf{d}$ the content of 18 glucosinolates by ULPC-MS methods. MeJA, methyl jasmonate. Se, sodium selenite. BR, brassinolide. GLS, glucosinolates. In Fig. $3 \mathrm{a}$ and $\mathrm{b},{ }^{*}$ and ${ }^{* *}$ notes $p<0.05$ and 0.01 vs. control, respectively, while \# and \#\# notes $p<0.05$ and $p<0.01$ vs. NaCl, respectively. In Fig. C, * notes $p<0.05$ vs. control, and \# notes $p<0.05$ vs. NaCl, respectively 
control, while MeJA increased both aliphatic and indolic glucosinolates, but decreased aromatic glucosinolates $(p$ $<0.01$, Fig. 1c). Figure 1d shows the influence of four treatments on the accumulation of 18 glucosinolates. We observed that BR showed slight influence on indolic glucosinolates, followed by aromatic glucosinolates and significantly obvious influence on aliphatic glucosinolates, including gluconapin, glucobrassicanapin, and glucoraphasatin (Fig. 1d); and MeJA showed obvious influence on most glucosinolates, including glucobrassicanapin, glucoraphanin, glucoraphasatin, n-hexyl- glucosinolates and glucobrassicin $(p<0.05)$.

\section{Summary of Illumina Hiseq and PacBio RSIl transcriptome sequencing data of Chinese kale}

Illumina Hiseq 2500 platform generated 816,939,038 raw reads and 799,683,878 clean reads $(97.89 \%, 119.97 \mathrm{G})$ with an average $\mathrm{Q}_{30}$ value of $93.46 \%$ and an average GC content of $47.05 \%$ (Table 1). A total of 130,553 unigenes with a 99.05\% annotation rate in at least one database [NR (NCBI non-redundant protein sequences), NT (NCBI non-redundant nucleotide sequences), Swissprot, Protein family (Pfam), Gene Ontology (GO), KOG (euKaryotic Orthologous Groups), KEGG (Kyoto Encyclopedia of Genes and Genomes) and KO (KEGG Ortholog database)], including 8006 transcription factors (TFs) were identified from Illumina transcriptome (Table 2). PacBio RSII platform generated a total of 617,063 circular consensus sequences (CCSs) with a full length of $467,672 \mathrm{bp}$. The full-length non chimera (FLNC) reads number was 434,967, with an average length of $1720 \mathrm{bp}$ (Table 2). PacBio RSII platform produced a total of 199,106 consensus reads and 14,267,901 subreads (13.46 G bases, with an average length of 944 bp, and an N50 of 1572 bp), which were then corrected using the Illumina reads (Additional file 1: Figure S1). The length distributions of gene (Additional file 2: Figure S2A and B), corrected subreads and consensus reads (Additional file 2: Figure S2C), lncRNA number (Additional file 2: Figure S2 S2D) and simple sequence repeat (SSR) motifs (Additional file 2: Figure S2E) are shown in Additional file 2: Figure S2. There was similar distribution of CDS of these unigenes produced by PacBio RSII platform and Illumina Hiseq 2500 platform, and most CDSs were less than 1800 bp (Additional file 2: Figure S2A and B). A total of 6369 long non-coding RNAs (lncRNAs) were identified from PacBio transcriptome (Additional file 2: Figure S2D).

In comparative analysis, we observed that the unigene number originated from PacBio RSII transcriptome (185,134 genes) was higher than that of Illumina transcriptome (130,553 genes; Table 1 and Fig. $2 \mathrm{a}$ and b), including 130,553 overlapping genes; the number of transfection factors (TFs) from PacBio RSII
Table 1 Summary of the transcriptome data from PacBio RSII platform and Illumina Hiseq platform

\begin{tabular}{|c|c|}
\hline Platform & \\
\hline Illumina Hiseq $(n=15)$ & \\
\hline Raw reads (average) & $5,562,602.5$ \\
\hline Clean reads (average) & $53,312,258.5$ \\
\hline Clean bases (G, total) & 119.97 \\
\hline Base error rate (average) & $0.015 \%$ \\
\hline Q30 (average) & $93.46 \%$ \\
\hline GC content (average) & $47.05 \%$ \\
\hline $\begin{array}{l}\text { Unigenes annotated in at least one database (NR, NT, KO, } \\
\text { Swissprot, Pfam, GO, KOG) }\end{array}$ & 129,325 \\
\hline Unigenes annotated in all databeses & 29,191 \\
\hline Total unigenes & 130,553 \\
\hline TF number & $8006(6.19 \%)$ \\
\hline PacBio RSII $(n=15)$ & \\
\hline Total CCS & 617,063 \\
\hline Full length reads & $\begin{array}{l}467,672 \\
(75.9 \%)\end{array}$ \\
\hline FLNC reads & $\begin{array}{l}434,967 \\
(70.5 \%)\end{array}$ \\
\hline Average FLNC read length & 1720 bp \\
\hline Consensus reads & 199,106 \\
\hline Subreads base (G) & 13.46 \\
\hline Subreads number & $14,267,901$ \\
\hline Average subreads length & $944 \mathrm{bp}$ \\
\hline N50 & 1572 \\
\hline $\begin{array}{l}\text { Total unigenes annotated in at least one database (NR, NT, KOG, } \\
\text { Swissprot, Pfam, GO, KEGG) }\end{array}$ & 140,196 \\
\hline Total unigenes & 44,938 \\
\hline TF number & $9407(5.08 \%)$ \\
\hline
\end{tabular}

CCS circular consensus sequences, FLNC full-length non chimera, GO Gene Ontology, KEGG Kyoto Encyclopedia of Genes and Genomes, KO KEGG Ortholog database, KOG euKaryotic Orthologous Groups, Nr NCBI nonredundant protein sequences, Nt NCBI non-redundant nucleotide sequences, Pfam Protein family, TF transcription factors

transcriptome was 9407, which was higher than 8006 from Illumina (Table 1; Fig. 2c and d).

\section{Functional annotation of unigenes}

The GO, KEGG, and KOG functional annotations of unigenes in PacBio RSII transcriptome were identical to unigenes in Illumina transcriptome (Additional file 3 Figure S3). GO classification showed most unigenes were associated with cellular and metabolic processes with the molecular functions of binding and catalytic activity (Additional file 3 Figure S3A). KEGG and KOG classification showed that the top clusters involved unigenes were energy and carbohydrate metabolism, transport, catabolism and signal transduction (Additional file 3 Figure S3B and C). Annotation against NR database showed that unigenes in PacBio transcriptome were identical to Brassica oleracea (48.4\%), followed with Brassica napus (38.0\%) and Brassica rapa (3.0\%), while 
Table 2 The primers' sequences of 22 genes for PCR analysis in this study

\begin{tabular}{|c|c|c|c|}
\hline \multirow[t]{2}{*}{ Genes ID } & \multirow[t]{2}{*}{ Gene and symbol } & \multicolumn{2}{|l|}{ Primers sequences $\left(5^{\prime}-3^{\prime}\right)$} \\
\hline & & Forward & Reverse \\
\hline CDX99400.1 & (cytosine-5)-methyltransferase CMT2 (DNMT2) & GAACGACGATGCTGAAGGTAG & CTGCTGCTGAGACTCTGACTA \\
\hline CDY40532.1 & S-adenosylmethionine synthase (SAMS) & AAGCCGCCACATAAGAGACTA & ACAGGACAAGAACAGACAGACT \\
\hline XP_009111749.1 & Uncharacterized & CCTGCCGTGTTGTCTTCC & GCTCTGTGGTTGTTCATCATCT \\
\hline CDY19364.1 & (cytosine-5)-methyltransferase CMT1 (DNMT1) & TGGATGGTGGAGATGCTTCA & CGTTCAAGGTCGTCGTCATT \\
\hline CDX85436.1 & 5-methyltetrahydropteroyltriglutamate--homocysteine methyltransferase 1 (metE) & ATGCTCGGTGCTGTTCCA & GCCTTGTGCGATGCGTAA \\
\hline CDX80735.1 & cytochrome P450 83 B1 (CYP83b1) & TTCACCGCTCGTCCTCTC & ACCATACACATCTTCCTCATCTCA \\
\hline CDX69373.1 & cytochrome P450 79B1 (CYP79b1) & TTCGGATCTCACTTCCACTACA & CATCACCAAGGTTATAGCCACAA \\
\hline CDY01177.1 & adenosylhomocysteinase 1 (SAHH) & CCGTCACCAAGAGCAAGTTC & CATCACCATATCCGCAGACAAC \\
\hline XM_009134853.1 & S-adenosylmethionine synthase 3-like (SAMS3L) & GCACGCCGCTGTATAGTC & ACACGAGTATATCCTTGTCTGGTA \\
\hline XP_009141614.1 & S-adenosylmethionine synthase 3-like (SAMS3L) & $\begin{array}{l}\text { GACA } \\
\text { AGACCATATTCCACCTCAAC }\end{array}$ & GCCTGCCTAACGATGTAAGC \\
\hline XP_009107632.1 & cytochrome P450 83B1 (CYP83b1) & $\begin{array}{l}\text { TGAG } \\
\text { ATGAGGAAGATGTGTATGGT }\end{array}$ & $\begin{array}{l}\text { ACAG } \\
\text { TTGGTGAATGACAAGAGAAG }\end{array}$ \\
\hline CDY12709.1 & cytochrome P450 83A1 (CYP83A1) & GGCGATGCTTGAGGTTCC & GCTTGAGATGATCCGACTTGTG \\
\hline XM_009138817.1 & methylthioalkylmalate synthase 2 (MAM2) & CTATCGTTATGGCTTCGTCACTTC & CGTAGGGAGGGCAAGGAA \\
\hline XM_009103756.1 & S-alkyl-thiohydroximate lyase SUR1 (SUR1) & TCTCACGACCATCTCCACAA & TCCAGCCAATCTTCCATCCA \\
\hline ACB59204.1 & UDP-glycosyltransferase 74B1 (UGT74B1) & CGACGCATACTCCGAATCC & GGTGAGGTTGTTGGTGAAGAA \\
\hline CDX75914.1 & S-adenosylmethionine synthase 2 (SAMS2) & CTGAGCCGTTGTCTGTGTT & ACCATAAGCAGCAGTCTTCAAG \\
\hline CDY37784.1 & S-adenosylmethionine synthase 2-like (SAMS2L) & GTTCAGGTCTCTTACGCCATTG & TGTCCATACGCAGCAGTCT \\
\hline DQ679980.1 & homocysteine S-methyltransferase 1 (HMT1) & TTGGTGATGCTGAGTTGGAGAT & TTGATGGTGGAAGGTGTAGTC \\
\hline CDX73052.1 & cytosolic sulfotransferase 16-like (SULT16L) & GGACACTGGTGGCAAGAATG & AGCGAGAACGGTTGACGATA \\
\hline XP_009106322.1 & adenosylhomocysteinase 2-like (SAHH2L) & GCTCACCAAGGACCAATCTG & ACCCGAACCAACTCAAACAAT \\
\hline AAP92453.1 & $\begin{array}{l}\text { adenosylhomocysteinase 1-like (LOC106311804), transcript variant X1 (SAHH1L- } \\
\text { X1) }\end{array}$ & GCTGGTGCTAGAGTCATTGTG & GCGTTGTTCTTCATCTTCCTCAT \\
\hline XP_009135865.1 & adenosylhomocysteinase 2 (LOC106335699), transcript variant X4 (SAHH2L-X4) & CGTGATGTCGTGCTCGTT & CTTCTCGTCCAAGTGCTTAGG \\
\hline XP_009114068.1 & S-adenosylmethionine synthase 2-like (SAMS2L) & GAGGTGGTAATGGTAGGTTCTTG & CTTGTTAGACTTGAGTGGCTTCA \\
\hline
\end{tabular}

the unigenes in Illumina transcriptome were identical to Brassica napus (63.0\%) followed with Brassica rapa (19.3\%; Additional file 3: Figure S3D).

\section{Identification of differentially expressed genes (DEGs) in response to different stimuli}

DEGs induced by different stimuli were identified from combined data of Illumina transcriptome with PacBio RSII transcriptome. A total of 6893 (including 3176 up- and 3717 down-regulated DEGs), 13,287 (including 4377 up- and 8910 down-regulated DEGs), 13,659 (including 4879 up- and 8780 down-regulated DEGs), and 11,041 DEGs (including 4351 up- and 6690 down-regulated DEGs) were identified from Chinese kale seedlings under $\mathrm{NaCl}$, Se, MeJA, and BR treatment, respectively ( $p$-value $\leq 0.05, \log 2 \mathrm{FC} \geq 1$ or $\leq-1$; Fig. 3a). A total of 2883 genes, including 685 up- and 2187 down-regulated DEGs, were simultaneously dysregulated by all $\mathrm{NaCl}$, Se (sodium selenite $100 \mu \mathrm{M})$, MeJA $(40 \mu \mathrm{M})$, and brassinolide (BR, $1.5 \mu \mathrm{M})$ treatments (Fig. 3b). The heatmap clustering of these DEGs is shown in Fig. 3c.

\section{GO and KEGG pathway enrichment analysis}

Functional enrichment analysis showed $\mathrm{NaCl}, \mathrm{BR}$, MeJA, and Se-induced DEGs were associated with 26 $\sim 135$ GO terms including biological process terms of indole-containing compound metabolic process, cellular glucan metabolic process, carbohydrate metabolic process, and polyol biosynthetic process; cellular component terms of Holliday junction resolvase complex and external encapsulating structure; and molecular function terms of hydrolase activity of hydrolyzing O-glycosyl compounds, calcium ion binding, xyloglucan-xyloglucosyltransferase activity, and polysaccharide metabolic process (Additional file 4: Table $\mathrm{S1}$ ). These DEGs were significantly involved in KEGG pathways including starch and sucrose metabolism, limonene and pinene degradation, stilbenoid, diarylheptanoid and gingerol biosynthesis, phenylalanine, tyrosine and tryptophan biosynthesis, valine, leucine and isoleucine biosynthesis, cysteine and methionine metabolism, and glucosinolate biosynthesis (Additional file 5: Table S2). The DEGs induced by MeJA, $\mathrm{BR}$, and Se treatments were associated with plant-pathogen interaction pathway, and Se-induced 


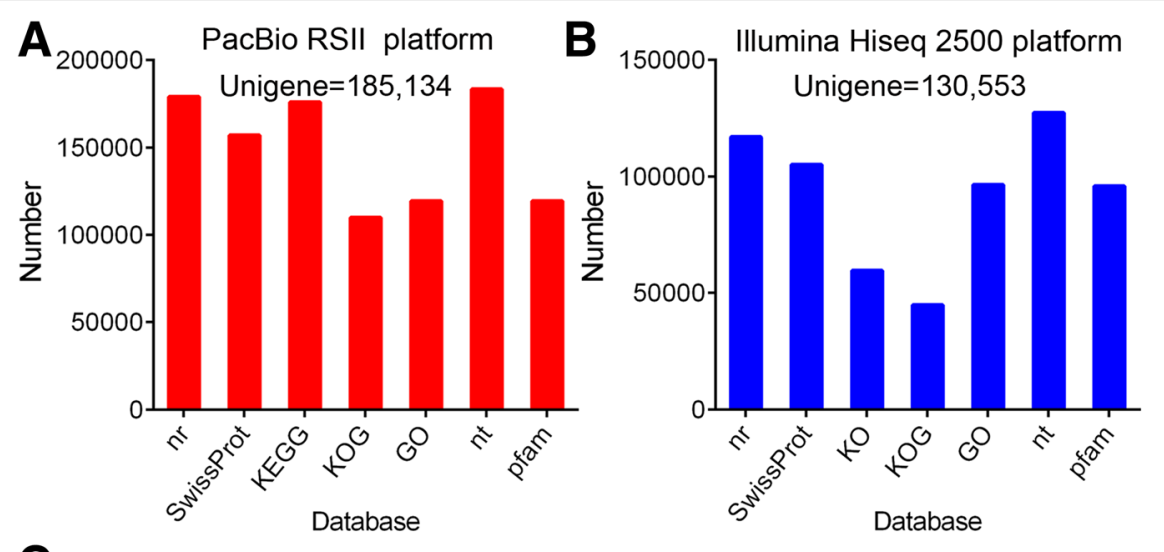

C
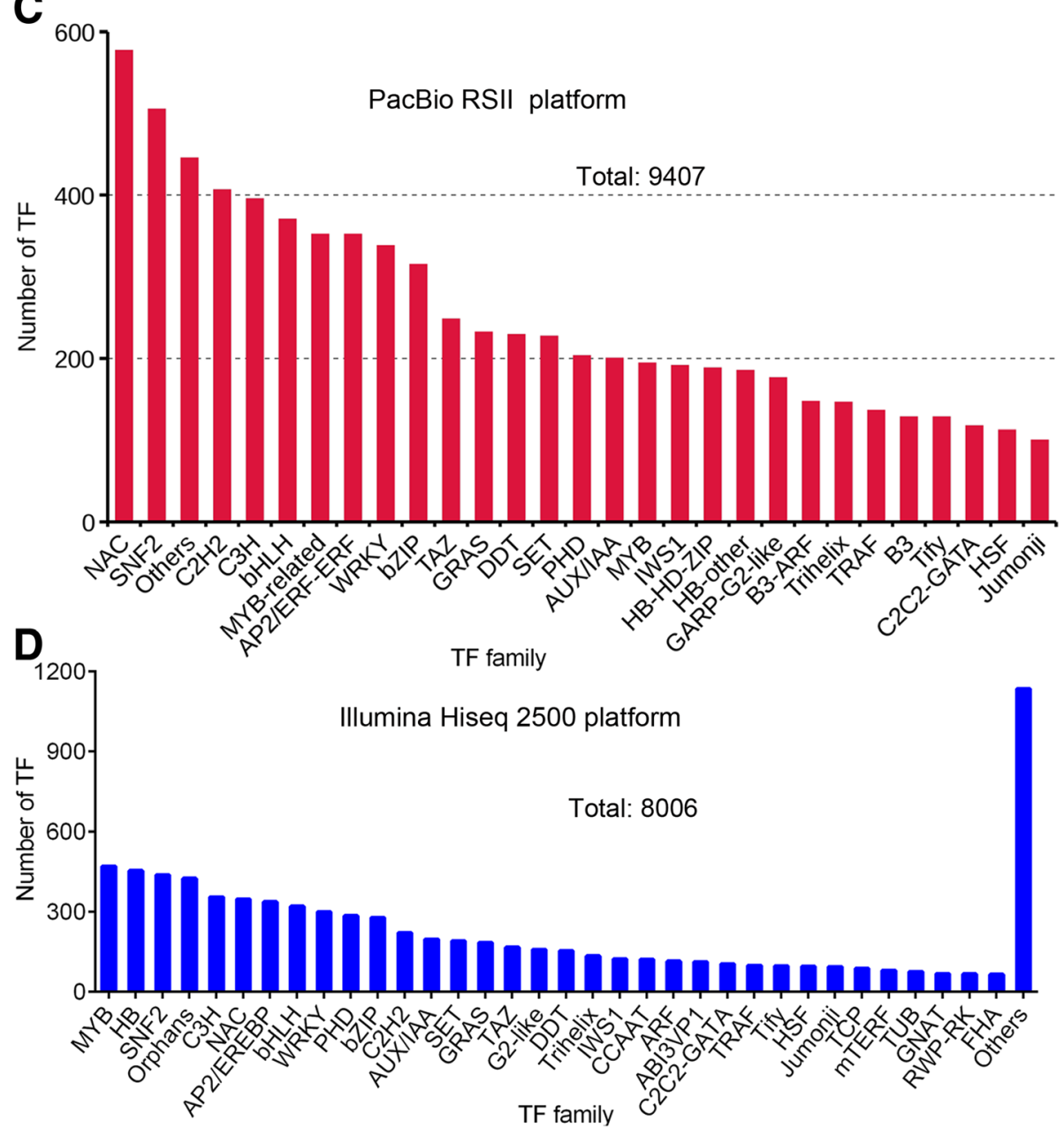

Fig. 2 The unigene and transcription factor (TF) numbers produced by different sequencing platforms. $\mathbf{a}$ and $\mathbf{b}$, the unigene numbers in transcriptome sequencing data generated from PacBio RSII platform and Illumina Hiseq sequencing platform, respectively. c and $\mathbf{d}$, the TF numbers in PacBio RSII and Illumina transcriptome, respectively

DEGs were associated with the biosynthesis of glucosinolates (Additional file 5: Table S2 and Additional file 6: Figure S4). The KEGG pathways associated with DEGs induced by four stimuli are shown in Additional file 5: Table S2.
Analysis of DEGs in pathways related to glucosinolate biosynthesis

According to aforementioned results, we confirmed that the influence of $\mathrm{NaCl}, \mathrm{BR}, \mathrm{MeJA}$, and Se treatments on glucosinolates accumulation might be mediated by 


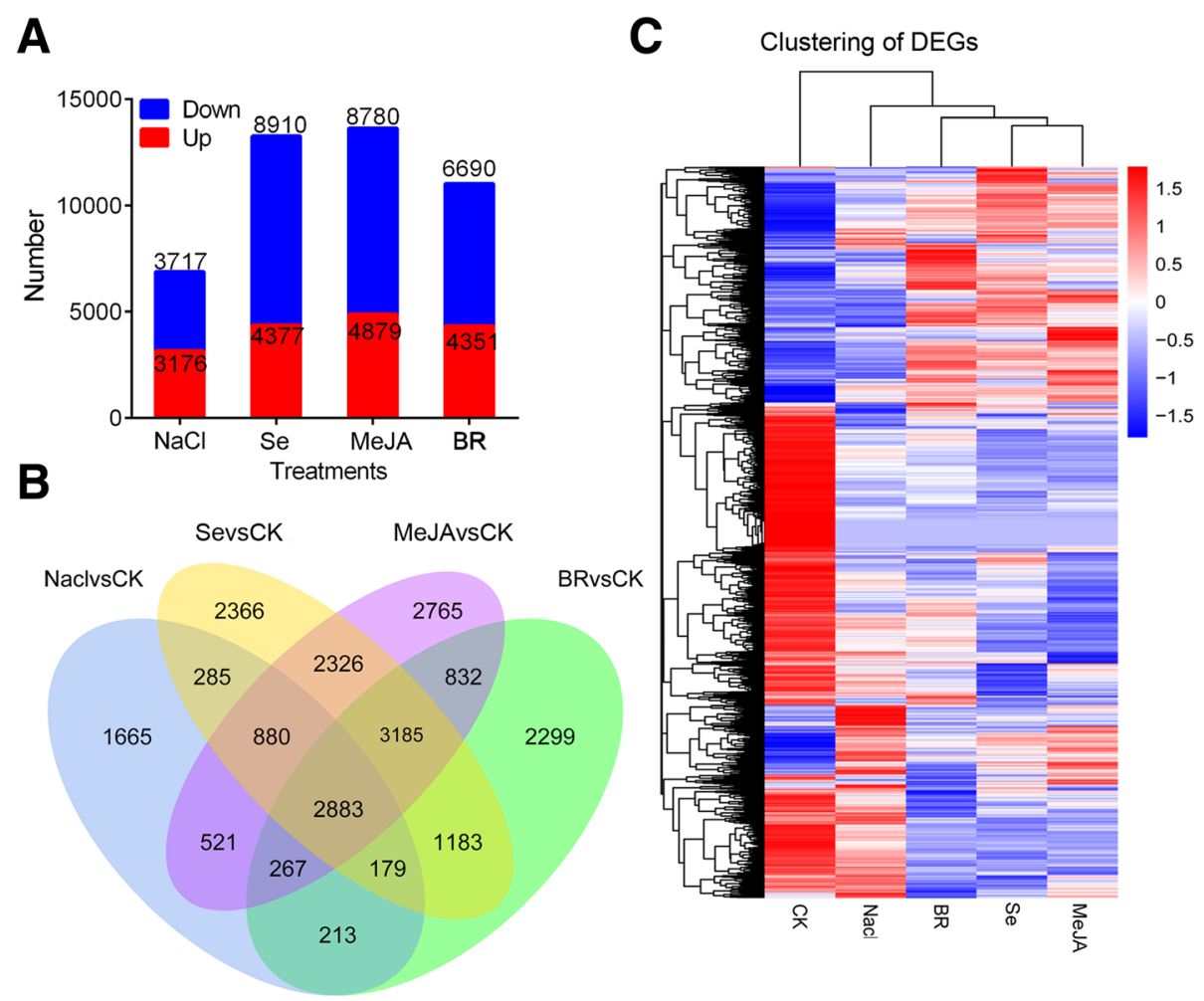

Fig. 3 The distribution of differentially expressed genes (DEGs) induced by different treatments. $\mathbf{a}$ and $\mathbf{b}$, the distribution of DEGs induced by different treatments and the venn figure, respectively. c clustering analysis of the DEGs. Blue, downregulated. Red, upregulated. MeJA, methyl jasmonate; Se, sodium selenite, BR, brassinolide

altering the expression profiles of sulforaphane and/or glucosinolate biosynthesis related genes. Accordingly, we found the FPKM (fragments per kilobase of transcript per million mapped reads) values of 23 unigenes related to glucosinolate biosynthesis were dysregulated by $\mathrm{NaCl}$, BR, MeJA, and Se treatments (Fig. 4a). The mRNA relative expression levels profiles of most genes by qRT-PCR were similar to that of FPKM values by sequencing (Fig. $4 a$ and $b)$.

Sequencing and qRT-PCR analysis confirmed the expressions of SAMS (S-adenosylmethionine synthase; CDY40532.1), SAHH (adenosylhomocysteinase 1; CDY01177.1) and HMT1 (homocysteine S-methyltransferase 1; DQ679980.1) were upregulated by $\mathrm{NaCl}$ treatment; the expression of DNMT1 [(cytosine-5)-methyltransferase CMT1; CDY19364.1], DNMT2 (CDX99400.1) and metE (5-methyltetrahydropteroyltriglutamate:homocysteinemethyltransferase 1; CDX85436.1) were increased by Se, MeJA and BR treatments; the expression of CYP83b1 (CDX80735.1 and XP_009107632.1), CYP79b1 (CDX69373.1), SUR1 (XM_009103756.1) and UDPglycosyltransferase 74B1 (UGT74b1; ACB59204.1) were upregulated by MeJA and BR ( $p<0.05$, Fig. $4 \mathrm{a}$ and b). Different expression pattern of SAMS3L (XP_009141614.1), CYP83b1 (XP_009107632.1), CYP83a1 (CDY12709.1),
MAM2 (methylthioalkylmalate synthase 2; XM_009138817.1), SAHH1L-X1 (adenosylhomocysteinase 1-like (LOC106311804), transcript variant X1; AAP92453.1) and SAHH2L X4 (adenosylhomocysteinase 2 (LOC106335699), transcript variant X4; XP_009135865.1) might due to the loss assembled reads by sequencing. The consistency between sequencing and PCR analyses of 17 genes $(73.91 \%, 17 / 23)$ showed the high confidence of sequencing data. The glucosinolate biosynthesis pathway and DEGs induced by $\mathrm{Se}, \mathrm{NaCl}, \mathrm{MeJA}$ and $\mathrm{BR}$ are shown in Additional file 6: Figure S4. The key genes involved in glucosinolate biosynthesis was the desulfoglucosinolate sulfotransferase (SOT) A/B/C (ST5a_b_c), which were downregulated by all treatments (Additional file 7: Figure S5), suggesting the impact of different treatment on glucosinolate biosynthesis.

\section{Dysregulated TFs}

We further confirmed that $\mathrm{NaCl}, \mathrm{MeJA}, \mathrm{BR}$ and $\mathrm{Se}$ treatment downregulated the members in MYB family (including MYB1R1, MYB44, WRKY45 and MYB24), AUX/IAA family (IAA9, IAA11 and auxin response factor 2, ARF2), NAC family (NAC-domain protein 5-11, NAC5-11; NAC55, ATAF2, and NAC29), AP2-EREBP family members (ERF19, ERF113, RAP2-13, and 


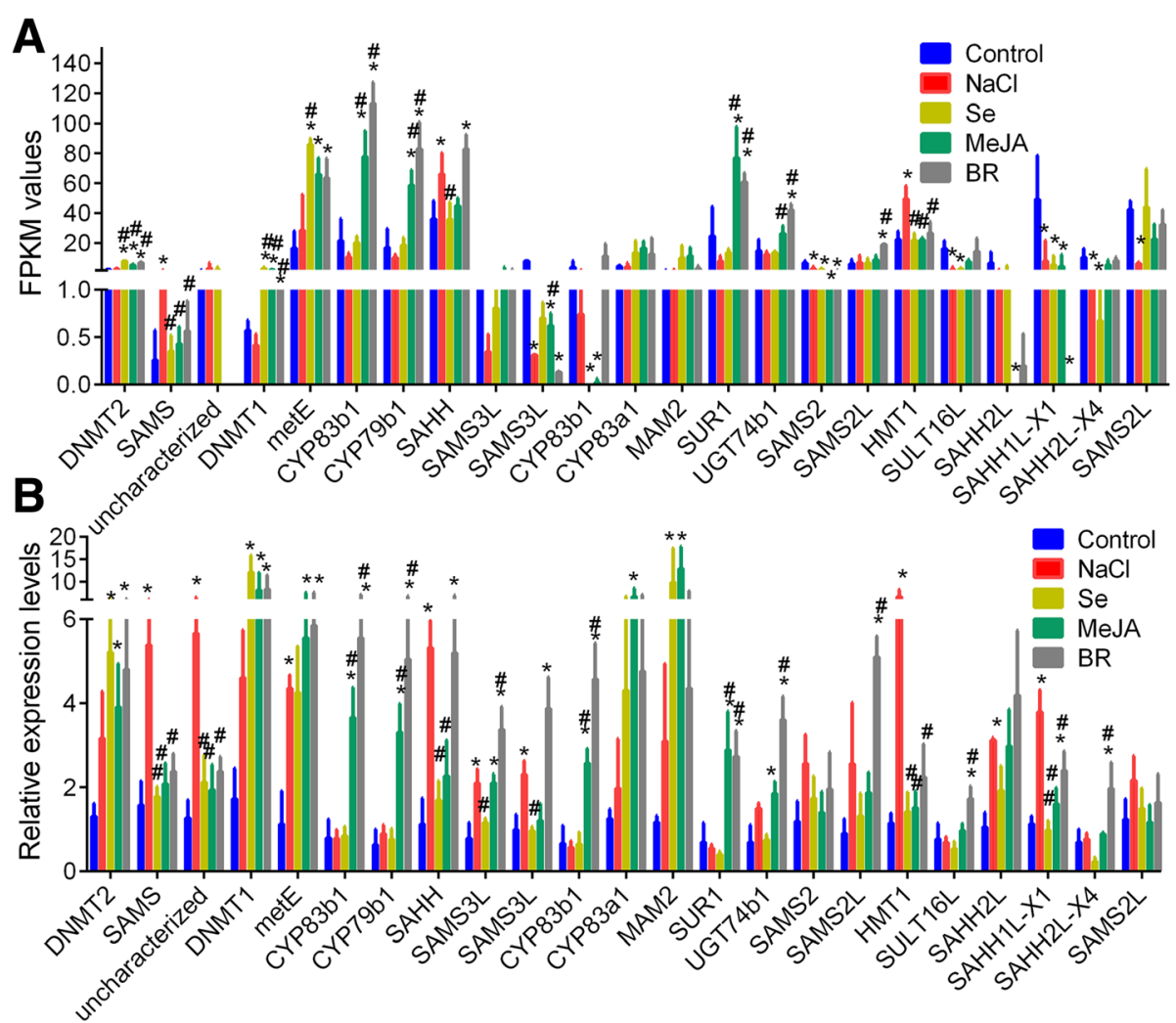

Fig. 4 The FKPM values and relative levels of 23 genes related to glucosinolate biosynthesis. a the FKPM values of 23 genes in samples treated with different treatments by sequencing. $\mathbf{b}$ the relative expression levels of 23 genes determined by qRT-PCR. ${ }^{*}$ notes $p<0.05$ vs. control, and \# notes $p<0.05$ vs. NaCl, respectively. MeJA, methyl jasmonate; Se, sodium selenite, BR, brassinolide

ERF12), SNF2 family, and WRKY family (including WRKY33, WRKY18, WRKY48, WRKY51, WRKY22-1), and upregulated MYB28 and WRKY47. Most TFs were downregulated by treatments (Fig. 5).

\section{Discussion}

Glucosinolates are a group of sulfur-rich anionic products naturally generated in widely consumed cruciferous vegetables. We performed the combined transcriptome analysis of NGS and SMRT sequencing and investigated the molecular mechanism of sulforaphane and glucosinolate biosynthesis in Chinese kale seedlings in response to abiotic stresses. The high error rate in long reads generated from PacBio platform was corrected by short reads produced by Illumina sequencing. We found SMRT transcriptome contained 185,134 unigenes, that was higher than 130,553 unigenes from Illumina transcriptome, suggesting higher performance of SMRT sequencing compared with NGS.

The combination of NGS short reads and SMRT long reads identified 6893, 13,287, 13,659 and 11,041 DEGs in kale seedlings in response to $\mathrm{NaCl}(75 \mathrm{mM})$, MeJA $(40 \mu \mathrm{M})$, Se $(100 \mu \mathrm{M})$ and BR $(1.5 \mu \mathrm{M})$ treatment, respectively. KEGG pathway enrichment analysis showed these genes were associated with energy metabolism, biosynthesis of amino acids including phenylalanine, tyrosine, tryptophan, phenylalanine, cysteine, methionine, valine, leucine and isoleucine, and glucosinolate (Additional file 5: Table S2). These pathways were core pathways for the synthesis of glucosinolates (Additional file 6: Figure S4). These facts showed that these biosynthetic pathways were influenced by $\mathrm{NaCl}, \mathrm{MeJA}$, Se and BR treatments in Chinese kale seedlings, and the glucosinolates might take important roles in the plant defense or resistance to these abiotic stresses.

In stressed or specific conditions, glucosinolates are released from vacuoles and are hydrolyzed by MYO to unstable thiohydroximate-O-sulfonate, which spontaneously converts to isothiocyanates including sulforaphane $[17,29]$. Glucosinolates are grouped into three groups, including aliphatic, aromatic and indolic glucosinolates. The tryptophan amino acid is the substrate of indolic glucosinolates, and methionine is the substrate of aliphatic glucosinolates [13, 17]. The subsequently conversion of tryptophan-derived indolic glucosinolates and methionine-derived aliphatic glucosinolates to S-alkylthiohydroximates (SAM) is catalyzed by CYP83b1 and CYP83a1 [30]. Next, SAMs are conversed into 


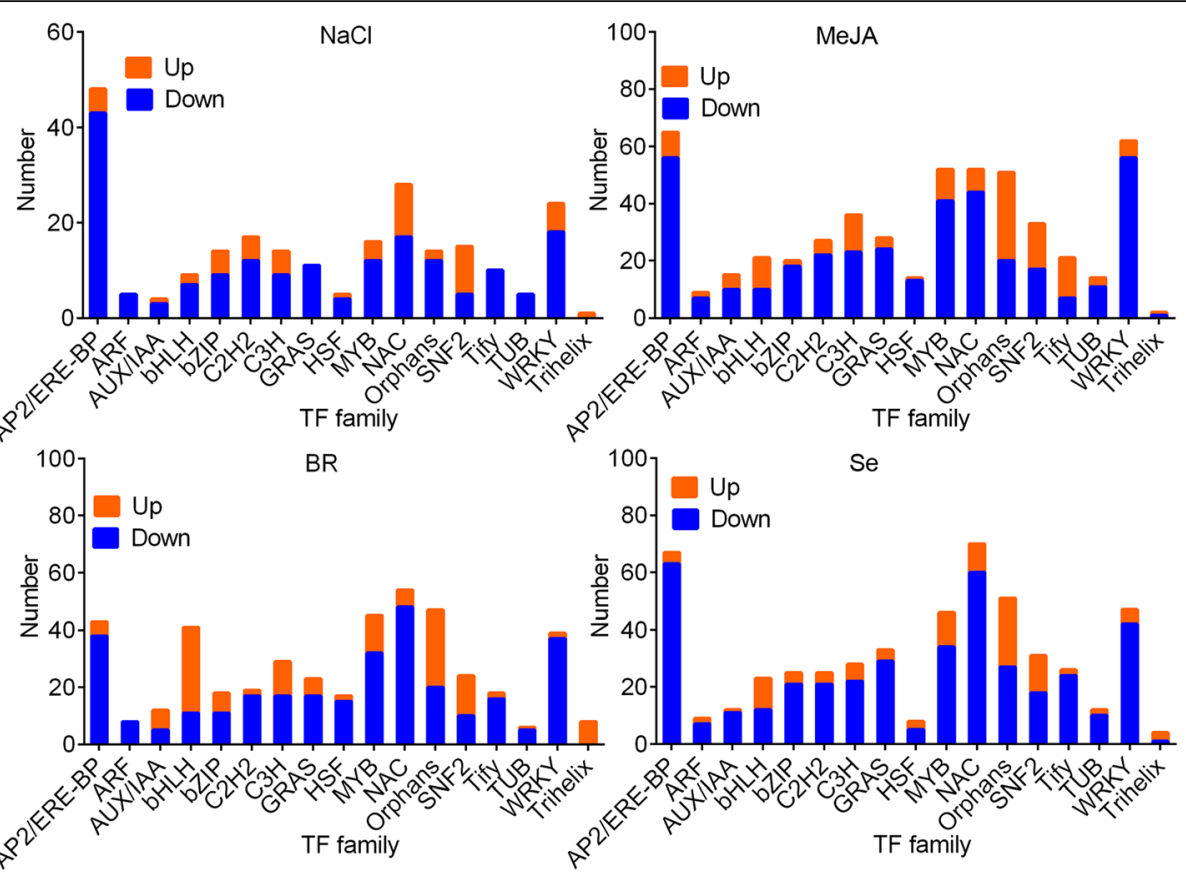

Fig. 5 Dysregualted transcription factor (TF) numbers by four different treatments. Blue, downregulated TFs. Orange, upregulated TFs

thiohydroximates, desulfoglucosinolates and sulfated products by SUR1, UGT74B1 and ST5b_c, respectively, in step-by-step ways [30-32]. We confirmed that the content of aliphatic glucosinolates was highest in Chinese kale seedlings, followed with indolic glucosinolates and then aromatic glucosinolates. In addition, we confirmed that the content of aliphatic glucosinolates was increased by MeJA and BR treatments, while aromatic glucosinolates was increased by Se and BR treatments (Fig. 3). Transcriptome analysis showed that the expression of genes in core pathways of aliphatic (including CYP83a1, SUR1, and UGT74b1) and aromatic glucosinolates (including CYP83b1, SUR1 and UGT74b1), were increased by MeJA and BR treatments (Fig. 4 and Additional file 6: Figure S4). In addition, we found the contents of sulforaphane and total glucosinolates were increased by MeJA and BR treatments compared with control. The expressions of CYP83b1, SUR1 and UGT74b1 were upregulated followed MeJA treatment (Fig. 4). Although $\mathrm{NaCl}$ treated decreased the content of aliphatic and indolic glucosinolates and sulforaphane, the expression of the aforementioned core genes did not decrease by $\mathrm{NaCl}$ treatment. This might due to the decreased expression of ST5a_b_c by $\mathrm{NaCl}$ treatment (Additional file 7: Figure S5). We also observed the decrement in ST5a_b_c expression by Se, MeJA and BR. It has been reported that the synthesis of aliphatic glucosinolates could be controlled by ST5b_c, and the aromatic glucosinolates is controlled by ST5a (Additional file 6: Figure S4). These demonstrated the complex and ordinal mechanism of glucosinolates biosynthesis in plant [33].

ST5a_b_c, are three desulfoglucosinolate SOTs that catalyze the final step in glucosinolate biosynthesis by catalyzing different substrate specificities and producing S-containing products $[34,35]$. The most important glucosinolate, sulforaphane, is a sulfur-rich hydrolysis product of aliphatic (methylseleno)glucosinolate, the methionine-derived glucosinolates [36-38]. Our current study confirmed that the contents of all glucosinolates and sulforaphane in Chinese kale were decreased by $\mathrm{NaCl}$ stress $(75 \mathrm{mM})$. This was consistent with the results from the study by Esfandiari et al. [17]. Esfandiari et al. showed that $\mathrm{NaCl}$ stress (80 and $160 \mathrm{mM}$ ) decreased the content of glucosinolates in broccoli via suppressing the expression of CYP83a1 and CYP83b1 [17]. They also suggested that the increased contents of sulforaphane by $\mathrm{NaCl}$ stress ( 80 and $160 \mathrm{mM}$ ) were associated with upregulation of MYO and ESM1. Guo et al. showed the higher expression of MYO was found in Chinese kale roots with low-glucosinolates [25], which might responsible for the increased sulforaphane [17]. Our study demonstrated that the expression of CYP83a1 and CYP83b1 were not significantly influenced by 75 $\mathrm{mM} \mathrm{NaCl}$ stress. In addition, the treatment with $75 \mathrm{mM}$ $\mathrm{NaCl}$ decreased the contents of both sulforaphane and glucosinolates. Although total and the major aliphatic glucosinolates were increased by MeJA and BR treatments, the FPKM of ST5a (crucial for aromactic glucosinolates) and ST5b_c (crucial for aliphatic 
glucosinolates; Additional file 6: Figure S4) coding sequences were decreased by MeJA and BR compared with control (Additional file 7: Figure S5). These differences might due to the plant variety, tissues or growth conditions [17, 27, 39].

Other studies of Chinese kale transcriptome showed that the expression of CYP83a1 and CYP83b1 showed high expression level in root [24], and in plants with low contents of glucosinolates [25]. It has been reported that the biosynthesis of glucosinolates in susceptible varieties of Chinese cabbage were active than that in resistant varieties, and glucosinolates were increased by infection of Plasmodiophora [39], while that in resistant varieties was not influenced by treatments. They also showed that the two susceptible varieties had different contents of indolic and aliphatic glucosinolates in leaves or roots, while aromatic glucosinolates were the major glucosinolates in resistant varieties in response to Plasmodiophora, which were differentially influenced by the addition of SA and JA [39]. Our study was performed with 7-day old (post germination) Chinese kale seedlings, and the whole plant contents of glucosinolates were detected. The distribution of glucosinolates was averaged then, and these might also be the reasons for the differences between our results with others from Ludwig-Müller et al. [40], Esfandiari et al. [17] and others.

\section{Conclusion}

In summary, we performed the de novo assembly of Chinese kale genomes using SMRT sequencing combined with NGS sequencing. DEGs induced by $\mathrm{NaCl}$, MeJA, BR and Se treatment were identified using combination of NGS and SMRT transcriptome. MeJA and BR treatments increased the content of total glucosinolates and/or sulforaphane by upregulated the expression of genes related to glucosinolate core pathways, including CYP83b1, CYP83b1, SUR1 and/or UGT74b1. We also confirmed that $\mathrm{NaCl}(75 \mathrm{mM})$ inhibited the biosynthesis of sulforaphane, tryptophan-derived indolic glucosinolates and methionine-derived aliphatic glucosinolates, and MeJA treatment enhanced them. We concluded that the treatment of MeJA, Se and BR, especially MeJA, confer the formation and biosynthesis of glucosinolates and sulforaphane.

\section{Methods}

\section{Plant materials and preparation}

High sulforaphane Chinese kale germplasm resource BOK92 (inbred line) was obtained from the seed bank of College of Horticulture and Landscape, Hunan Agricultural University, Changsha, China. Seeds were sawed in nutrient soils (silica sands, sermiculite). After germination, seedlings were managed with standard nutrient solutions and treated with $\mathrm{NaCl}(0,25,50,75,100,125$ and $150 \mathrm{mM}), \operatorname{MeJA}(0,20,40,60,80,100$ and $120 \mu \mathrm{M})$, Se $(0,20,40,60,80,100$ and $120 \mu \mathrm{M})$ and BR $(0.0,0.5$, 1.0, 1.5, 2.0, 2.5 and $3.0 \mu \mathrm{M}$ ) with four triplicates of 30 seeds each. Control group was treated with standard nutrient solutions only. Experiments were performed in tissue culture room of Hunan Agricultural University at $22{ }^{\circ} \mathrm{C} \sim 25^{\circ} \mathrm{C}, 75 \%$ relative humidity, $35,000 \mathrm{~lx}$ illumination intensity, and a 16 L: $8 \mathrm{D}$ light cycle, with two handle irradiation per day. At 7-day post germination, seedlings were collected, snapped in liquid nitrogen, and stored at $-80^{\circ} \mathrm{C}$ before RNA isolation.

\section{Extraction and measurements of sulforaphane and glucosinolate}

Sulforaphane was extracted from frozen samples of kale seedlings. Samples were grinded into powder and then immersed into purified water for $2 \mathrm{~h}$ at room temperature, followed with dichloromethane extraction for $10 \mathrm{~min}$. Sodiumfulfate was used for drying. Extractions were then firstly filtered, vacuum dried, eluted using acetonitrile, and finally filtered $(0.22 \mu \mathrm{m})$. Sulforaphane standard was purchased from Shanghai yuanye Bio-Technology, China, (CAS No. 4478-93-7, Art. No.G18O8C45982). The content of sulforaphane was determined using high performance liquid chromatography (HPLC: Shimadzu, Kyoto, Japan) methods with conditions as follows: C18 column $(250 \mathrm{~mm} \times 4.6 \mathrm{~mm}$, $5 \mu \mathrm{m}$, at $\left.30{ }^{\circ} \mathrm{C}\right)$, mobile phase $(20 \%$ acetonitrile- $80 \%$ water) at speed of $1 \mathrm{~mL} / \mathrm{min}$, retention time $20 \mathrm{~min}$, and wavelength $202 \mathrm{~nm}$.

Glucosinolate were methanol-extracted from kale seedlings. Grinded powder was extracted with $70 \%$ methanol at $75^{\circ} \mathrm{C}$ for $20 \mathrm{~min}$, with addition of sinigrin (in water, $0.5 \mathrm{mg}$, internal standard). After the glucosinolate extraction became cold, barium acetate was added, followed with centrifugation. The supernatants were then extracted with $70 \%$ methanol and centrifuged for supernatants. Desulfoglucosinolates were isolated using anion-exchange chromatography. DEAESephadex A-25 was pretreated with acetic acid and then filled with resin. Crude extract was eluted with water and desulfoglucosinolates were eluted using sulfatase solution at room temperature overnight. Desulfitation glucosinolates were collected using water-elution. Desulfoglucosinolates were separated using chromatography with gradually increased methanol concentrations [5\% methanol for 1 min, linear gradient methanol (5\% 100\%) for $8 \mathrm{~min}$, $100 \%$ methanol for $2 \mathrm{~min}$, and $5 \%$ methanol for $2 \mathrm{~min}$ ]. The contents of desulfoglucosinolates were determined using ULPC-MS technology [diode array detector (229 $\mathrm{nm})$, C18 column $(100 \times 2.1 \mathrm{~mm})$, mobile phase of $75 \%$ methanol-25\% water] and mass spectrometer. 
Glucosinolates standards were purchased from (Sigma-Aldrich, GER, CAS No. 3952-98-5, Art. No. S1647-500MG).

\section{RNA extraction and transcriptome sequencing}

Altered genomic expression profiles in response to different stimulation $(\mathrm{NaCl}, \mathrm{MeJA}, \mathrm{SE}$ and $\mathrm{BR})$ were identified using the NGS. Total RNA was isolated from kale seedlings using TRIzol (Invitrogen, USA) and treated with RNase-free DNase I (Takara, Japan). After quality assessment using Agilent 2100 Bioanalyzer (Agilent Technologies, USA) and NanoDrop2000c spectrophotometer (NanoDrop products, USA), RNA samples were used for the synthesis of the first strand cDNA using a SMARTer PCR cDNA Synthesis Kit (Clontech Laboratories, USA). PCR optimization followed with large scale PCR were conducted for Illumina sequencing (the NGS, Hiseq PE 150) and the PacBio RSII sequencing (the third generation sequencing). Fifteen libraries were constructed and subjected to the NGS using Illumina sequencing platform and third-generation sequencing using the Pacific Biosciences RSII sequencing platform.

\section{Data processing}

The NGS data were collected from Illumina sequencing platform and processed as usual. Data quality estimators, including base error rate (\%), phred score $\left(\mathrm{Q}_{30}\right), \mathrm{GC}$ content (\%), and clean data rate (\%), was evaluated. Sequence data (CCS) containing full length and non-full length reads were generated from PacBio RSII platform and were processed using the SMRTlink 4.0 software ( $\min$ Passes $=1$, min Predicted Accuracy $=0.8)$. Non-full length and full-length fasta files were then fed into the cluster step (isoform-level clustering, ICE). Hiseq reads in full-length transcripts were adjusted using proovread software [41] and used as the reference sequences in this study. Redundancy in the adjusted transcripts was removed using CD-HIT-EST program and unigenes were obtained subsequently.

\section{Gene functional annotation, CDS predication, analysis or SNP and SSR}

$\mathrm{Nr}$, Nt, Pfam, KEGG, KOG, GO, Swiss-Prot, and KO databases were visited for gene function of the unigenes. Open reading frame (ORF) corresponded to the unigenes were identified with basting against NR and Swissprot proteins databases. Estscan software (v3.0.3) [42] was used for remedial prediction of ORF in unigenes. Single nucleotide polymorphisms and insertion-deletions of the transcriptome were called using GATK3 (v3.2, $\mathrm{QUAL}<30.0$ and $\mathrm{QD}<5.0)$ [43]. SSRs of the transcriptome were identified using MISA (v 1.0, http://pgrc.ipkgatersleben.de/misa/misa.html).

\section{Identification of DEGs}

Clean reads assembled using Trinity (version trinityrnaseq-2.0.2) [44], and assembled transcriptome was used for reads mapping by RSEM software [45]. Accordingly, FPKM levels of unigenes were obtained and used for calculation of relative expression level. DEGs were identified using the DESeq $\mathrm{R}$ package (v1.10.1) [46], with the criteria of adjusted $p$-value $\leq 0.05$, and log2 (fold change) $\geq 1$ (up) or $\leq-1$ (down). Cluster analysis of DEGs was performed using heatmap clustering.

\section{GO and KEGG pathway enrichment analysis}

GOseq analysis (http://www.geneontology.org/) was conducted for GO functional classification analysis [47]. The KEGG pathways associated with DEGs were identified using KEGG-Based Annotation System (KOBAS) server (http://kobas.cbi.pku.edu.cn/home.do) [48]. Called GO terms and KEGG pathways with corrected $p$ value $(\mathrm{BH})<0.05$ were considered as significantly items.

\section{Identification of TFs and IncRNAs}

TF among the DEGs were predicted using iTAK software (v1.2). LncRNAs of the transcriptome were identified using Coding-Non-Coding-Index (CNCI, default parameters), Coding Potential Calculator (CPC, e-value $\leq 1 \mathrm{e}-10$ ), and Pfam database (default parameters).

\section{QRT-PCR analysis}

Isolated total RNA from kale seedlings were reversely transcribed into the first-strand cDNA using Bestarq PCR RT Kit (DBI Bioscience, Germany). Primers were synthesized by Sangon (Shanghai, China) and were listed in Table 2. DNA template $(1 \mu \mathrm{g})$ was used for PCR amplification using specific primers synthesized by Sangon (Shanghai, China, Table 1). Amplification $(20 \mu \mathrm{L})$ was performed using Sybr Green qPCR master Mix (GENEray, GK8020) on an ABI 7500 fast real time PCR machine (ABI, USA). The reaction conditions were as follows: $94{ }^{\circ} \mathrm{C}$ for $3 \mathrm{~min}, 40$ cycles of $94{ }^{\circ} \mathrm{C} 20 \mathrm{~s}, 58^{\circ} \mathrm{C} 20$ $\mathrm{s}, 72^{\circ} \mathrm{C} 20 \mathrm{~s}$. Relative expression levels of genes were analyzed using $2^{-\triangle \Delta C t}$ methods. The $18 \mathrm{~s}$ gene was used as internal normal control gene in this study.

\section{Statistical analysis}

All data of qRT-PCR analysis and contents of sulforaphane and glucosinolate were presented as mean \pm standard deviation. All experiments were repeated at least three times. Statistics were performed using GraphPad Prism 6.0. One-way ANOVA was used to compare the data between groups with Holm-Sidak post hoc test. $P<$ 0.05 was considered statistically significant. 


\section{Additional files}

Additional file 1: Figure S1. Comparison of PacBiosubreads quality and corrected reads. (TIF $1683 \mathrm{~kb}$ )

Additional file 2: Figure S2. The analysis of the length distribution of CDS, PacBio reads and IncRNA number. $A$, the length distribution of blast and Estscan-predicted CDS in unigenes in Illumina sequencing data, respectively. B and C, the length distribution of blast CDS in unigenes, Subreads, and consensus reads in PacBio sequencing data, respectively. D, the venn figure of IncRNA number predicted in different softwares. E and $F$, the distribution of SSR motifs in transcriptome generated from different platform. (TIF $1145 \mathrm{~kb}$ )

Additional file 3: Figure S3. Gene functional annotation of unigenes in transcriptome data from different platform. A-D, GO, KEGG, KOG, and NR classification of unigenes in transcriptome data from PacBio platform (Left) and Illumina (Right), respectively. (TIF 1729 kb)

Additional file 4: Table S1. Significant GO classifications associated with dfifferentially expressed genes (DEGs) induced by different treatments. (XLSX $754 \mathrm{~kb}$ )

Additional file 5: Table S2. Significant KEGG pathways associated with dfifferentially expressed genes (DEGs) induced by different treatments. (XLSX $72 \mathrm{~kb}$ )

Additional file 6: Figure S4. The glucosinolate biosynthesis pathway and related DEGs induced by sodium selenite treatment. Red and yellow boxes indicate upregulated DEGs, and green notes downregulated DEGs. (TIF $2356 \mathrm{~kb}$ )

Additional file 7: Figure S5. The FPKM level of ST5a_b_c under different treatments. ${ }^{*}$ and ${ }^{* *}$ notes $p<0.05$ and 0.01 vs. control, respectively. \# notes $p<0.05$ vs. $\mathrm{NaCl}$. (JPG $80 \mathrm{~kb}$ )

\section{Abbreviations}

BR: Brassinolide; CCS: Circular consensus sequences; CNCl: Coding-NonCoding-Index; CPC: Coding potential calculator; DEG: Differentially expressed genes; FLNC: Full-length non chimera; GO: Gene Ontology;

GT: Glucuronyltransferase; IL-1 $\beta$ : Interleukin-1 $\beta$; KEGG: Kyoto Encyclopedia of genes and genomes; KOG: Clusters of orthologous groups of proteins; LncRNAs: Long non-coding RNA; MAMs: Methylthioalkylmalate synthases; MeJA: Methyl jasmonate; NGS: Next-generation sequencing; NR: NCBI nonredundant protein sequences; Nrf2: Nuclear factor erythroid 2-related factor 2; NT: NCBI non-redundant nucleotide sequences; ORF: Open reading frame; SA: Salicylic acid; SAM: S-alkylthiohydroximates; Se: Sodium selenite; SMRT: Single-molecule real-time; SSR: Simple sequence repeat; SUR1: S-alkylthiohydroximatelyase or carbon-sulfur lyase; TF: Transcription factors: UGT74b1: UDP-glycosyltransferase 74B1

\section{Acknowledgements}

We deeply thank Prof. Jingui Zheng (Fujian Agricultural and Forestry University) and Prof. Jiashu Cao (Zhejiang University) for their valuable comments and discussion on previous versions of the manuscript. We are also grateful to anonymous reviewers for their helpful suggestions and comments.

\section{Funding}

This work was supported byNational Natural Science Foundation of China (No. 31772325), Hunan Provincial Natural Science Foundation of China (2017JJ2118,2018JJ3217), Hunan Provincial Sci-Tech Project (2018NK2022) and Scientific Research Fund of Hunan Provincial Education Department (16A093). The funders did not have any role in the design, collection, analysis, and interpretation of data and in writing the manuscript.

\section{Availability of data and materials}

All the data was available in present paper. The sequencing data was available at SRA database with accession number of SRP188508.

\section{Authors' contributions}

QYW summaried the transcriptome sequencing data, annotated the unigenes function, performed the data analysis and prepared the original manuscript. JWW identified the DGs, attended discussion and revised MS.
SXM performed the sulforaphane and glucosinolates content measure and attended data re-interpreting discussion. HRX, QW, MTL, YMY, MYL prepared the plant material and attended the transcriptomes data analyses and discussion. $\mathrm{KH}$ designed the experiments, provided research platform, performed the pathway enrichment analysis, and revised the manuscript. All authors approved the final manuscript.

Ethics approval and consent to participate

Not required.

\section{Consent for publication}

Not applicable

\section{Competing interests}

The authors declare that they have no competing interests.

\section{Publisher's Note}

Springer Nature remains neutral with regard to jurisdictional claims in published maps and institutional affiliations.

Received: 6 November 2018 Accepted: 2 May 2019

Published online: 14 May 2019

\section{References}

1. Fahey JW, Talalay P. Antioxidant functions of sulforaphane: a potent inducer of phase II detoxication enzymes. Food Chem Toxicol. 1999;37(9-10):973-9.

2. Gametpayrastre L, Li P, Lumeau S, Cassar G, Dupont MA, Chevolleau S, Gasc $\mathrm{N}$, Tulliez J, Tercé F. Sulforaphane, a naturally occurring Isothiocyanate, induces cell cycle arrest and apoptosis in HT29 human Colon Cancer cells. Cancer Res. 2000;60(5):1426-33.

3. Heiss E, Herhaus C, Klimo K, Bartsch H, Gerhäuser C. Nuclear factor kappa B is a molecular target for sulforaphane-mediated anti-inflammatory mechanisms. J Biol Chem. 2001:276(34):32008-15.

4. Kanematsu S, Yoshizawa K, Uehara N, Miki H, Sasaki T, Kuro M, Lai YC, Kimura A, Yuri T, Tsubura A. Sulforaphane inhibits the growth of KPL-1 human breast cancer cells in vitro and suppresses the growth and metastasis of orthotopically transplanted KPL-1 cells in female athymic mice. Oncol Rep. 2011;26(3):603-8.

5. Liu H, Talalay P. Relevance of anti-inflammatory and antioxidant activities of exemestane and synergism with sulforaphane for disease prevention. Proc Natl Acad Sci U S A. 2013;110(47):19065-70

6. Fernandes $\mathrm{RO}$, Bonetto $\mathrm{JH}$, Baregzay $\mathrm{B}$, de Castro $\mathrm{AL}$, Puukila S, Forsyth $\mathrm{H}$ Schenkel PC, Llesuy SF, Brum IS, Araujo AS. Modulation of apoptosis by sulforaphane is associated with PGC-1 伪 stimulation and decreased oxidative stress in cardiac myoblasts. Mol Cell Biochem. 2014;401(1-2):6170.

7. Brooks JD, Paton VG, Vidanes G. Potent induction of phase 2 enzymes in human prostate cells by sulforaphane. Cancer Epidemiol Biomark Prev. 2001 10(9):949-54.

8. Balendiran GK, Dabur R, Fraser D. The role of glutathione in cancer. Cell Biochem Funct. 2010;22(6):343-52.

9. Rajendran P, Dashwood WM, Li L, Kang Y, Kim E, Johnson G, Fischer KA, Löhr CV, Williams DE, Ho E. Nrf2 status affects tumor growth, HDAC3 gene promoter associations, and the response to sulforaphane in the colon. Clin Epigenetics. 2015;7(1):102.

10. An YW, Jhang KA, Woo SY, Kang JL, Chong YH. Sulforaphane exerts its antiinflammatory effect against amyloid- $\beta$ peptide via STAT-1 dephosphorylation and activation of $\mathrm{Nrf} / \mathrm{HO}-1$ cascade in human THPmacrophages. Neurobiol Aging. 2016:38:1-10.

11. Lekcharoenkul P, Tanongkankit Y, Chiewchan N, Devahastin S. Enhancement of sulforaphane content in cabbage outer leaves using hybrid drying technique and stepwise change of drying temperature. J Food Eng. 2014; 122(1):56-61.

12. Gamet-Payrastre L, Lumeau S, Gasc N, Cassar G, Rollin P, Tulliez J. Selective cytostatic and cytotoxic effects of glucosinolates hydrolysis products on human colon cancer cells in vitro. Anti-Cancer Drugs. 1998:9(2):141.

13. Sánchez-Pujante PJ, Borja-Martínez M, Pedreño MÁ, Almagro L. Biosynthesis and bioactivity of glucosinolates and their production in plant in vitro cultures. Planta. 2017;246(1):1-14. 
14. Hanschen FS, Evelyn L, Monika S, Sascha R. Reactivity and stability of glucosinolates and their breakdown products in foods. Angew Chem. 2015; 53(43):11430-50

15. Guo RF, Yuan GF, Wang QM. Effect of $\mathrm{NaCl}$ treatments on glucosinolate metabolism in broccoli sprouts. J Zhejiang Univ Sci B(Biomedicine \& Biotechnology. 2013;14(2):124-31.

16. Song YK, Cha KH, Song DG, Chung D, Pan CH. Amplification of sulforaphane content in red cabbage by pressure and temperature treatments. J Korean Soc Appl Biol Chem. 2011;54(2):183-7.

17. Esfandiari A, Saei A, Mckenzie MJ, Matich AJ, Babalar M, Hunter DA. Preferentially enhancing anti-cancer isothiocyanates over glucosinolates in broccoli sprouts: how $\mathrm{NaCl}$ and salicylic acid affect their formation. Plant Physiol Biochem. 2017;115:343.

18. Conaway CC, Getahun SM, Liebes LL, Pusateri DJ, Topham DK, Botero-

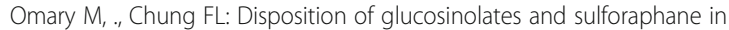
humans after ingestion of steamed and fresh broccoli. Nutr Cancer 2000, 38(2):168-178.

19. Kim SJ, Park WT, Uddin MR, Kim YB, Nam SY, Jho KH, Park SU. Glucosinolate biosynthesis in hairy root cultures of broccoli (Brassica oleracea var. italica). Nat Prod Commun. 2013;8(2):217-20.

20. Zang Y, Zhang H, Huang L, Wang F, Gao F, Lv X, Yang J, Zhu B, Hong SB, Zhu Z. Glucosinolate enhancement in leaves and roots of pak choi Brassica rapa ssp. chinensis ) by methyl jasmonate. Hortic Environ Biotechnol. 2015;56(6):830-40

21. Huang K, Lin JC, Wu QY, Yan JY, Liu MY, Zhang S, Xiao WJ. Changes in Sulforaphane and Selenocysteine methyltransferase transcript levels in broccoli treated with sodium selenite. Plant Mol Biol Report. 2015;34(4):1-8.

22. Tian M, Yang Y, Avila FW, Fish T, Yuan H, Hui M, Pan S, Thanhauser T, Li L. Effects of selenium supplementation on glucosinolate biosynthesis in broccoli. J Agric Food Chem. 2018;66(30):8036-44.

23. Kang-Mo K, Becker TM, Juvik JA. Transcriptome and metabolome analyses of Glucosinolates in two broccoli cultivars following Jasmonate treatment for the induction of Glucosinolate defense toTrichoplusia ni(Hübner). Int J Mol Sci. 2016;17(7):1135

24. Wu S, Lei J, Chen G, Chen H, Cao B, Chen C. De novo transcriptome assembly of Chinese kale and global expression analysis of genes involved in Glucosinolate metabolism in multiple tissues. Front Plant Sci. 2017:8:92.

25. Guo R, Huang Z, Deng Y, Chen X, Xu XH, Lai Z. Comparative transcriptome analyses reveal a special Glucosinolate metabolism mechanism inBrassica alboglabraSprouts. Front Plant Sci. 2016;7:1497.

26. Jeon J, Bong SJ, Park JS, Park YK, Arasu MV, Aldhabi NA, Park SU. De novo transcriptome analysis and glucosinolate profiling in watercress (Nasturtium officinaleR. Br.). BMC Genomics. 2017;18(1):401.

27. Guo RF, Yuan GF, Wang QM. Effect of $\mathrm{NaCl}$ treatments on glucosinolate metabolism in broccoli sprouts. J Zhejiang Univ B. 2013;14(2):124-31.

28. Jiao WB, Schneeberger $K$. The impact of third generation genomic technologies on plant genome assembly. Curr Opin Plant Biol. 2017;36:64-70.

29. Fahey JW, Zalcmann AT, Talalay P. The chemical diversity and distribution of glucosinolates and isothiocyanates among plants. Phytochemistry. 2001; 56(1):5-51.

30. Bak S, Feyereisen R. The involvement of two P450 enzymes, CYP83B1 and CYP83A1, in auxin homeostasis and Glucosinolate biosynthesis. Plant Physiol. 2001;127(1):108.

31. Gachon CMM, Langloismeurinne M, Henry Y, Saindrenan P. Transcriptional co-regulation of secondary metabolism enzymes in Arabidopsis: functional and evolutionary implications. Plant Mol Biol. 2005;58(2):229-45.

32. Mikkelsen MD, Naur $P$, Halkier BA. Arabidopsis mutants in the C-S lyase of glucosinolate biosynthesis establish a critical role for indole-3-acetaldoxime in auxin homeostasis. Plant J. 2010;37(5):770-7.

33. Markus P, Andreas S, Anna L, Axel M, Tim J, Weiler EW, Claudia O. Desulfoglucosinolate sulfotransferases from Arabidopsis thaliana catalyze the final step in the biosynthesis of the glucosinolate core structure. J Biol Chem. 2004:279(49):50717.

34. Klein M, Reichelt M, Gershenzon J, Papenbrock J. The three desulfoglucosinolate sulfotransferase proteins in Arabidopsis have different substrate specificities and are differentially expressed. FEBS J. 2010;273(1): 122-36.

35. Mckenzie MJ, Chen R, Leung S, Joshi S, Rippon PE, Joyce NI, Mcmanus MT. Selenium treatment differentially affects sulfur metabolism in high and low glucosinolate producing cultivars of broccoli (Brassica oleracea L). Plant Physiol Biochem. 2017;121:176.
36. Padilla G, Cartea ME, Velasco P, Haro AD, Ordás A. Variation of glucosinolates in vegetable crops of Brassica rapa. Phytochemistry. 2007; 68(4):536-45.

37. Zang YX, Kim JH, Park YD, Kim DH, Hong SB. Metabolic engineering of aliphatic glucosinolates in Chinese cabbage plants expressing Arabidopsis MAM1, CYP79F1, and CYP83A1. BMB Rep. 2008:41(6):472.

38. Matich AJ, Mckenzie MJ, Lill RE, Mcghie TK, Ronan K-YC, Rowan DD. Distribution of selenoglucosinolates and their metabolites in brassica treated with sodium selenate. J Agric Food Chem. 2015;63(7):1896-905.

39. Ludwig-Müller J, Schubert B, Pieper K, Ihmig S, Hilgenberg W. Glucosinolate content in susceptible and resistant chinese cabbage varieties during development of clubroot disease. Phytochemistry. 1997;44(3):407-14.

40. Sodhi YS, Mukhopadhyay A, Arumugam N, Verma JK, Gupta V, Pental D, Pradhan AK. Genetic analysis of total glucosinolate in crosses involving a high glucosinolate Indian variety and a low glucosinolate line of Brassica juncea. Plant Breed. 2010;121(6):508-11.

41. Hackl T, Hedrich R, Schultz J, Förster F. Proovread: large-scale high-accuracy PacBio correction through iterative short read consensus. Bioinformatics. 2014;30(21):3004-11

42. Iseli C, Jongeneel CV, Bucher P. ESTScan: a program for detecting, evaluating, and reconstructing potential coding regions in EST sequences. In ISMB. 1999:138-48.

43. Auwera GA, Carneiro MO, Christopher H, Ryan P, Guillermo DA, Ami LM, Tadeusz J, Khalid S, David R, Joel T. From FastQ data to high-confidence variant calls: the genome analysis toolkit best practices pipeline. Curr Protoc Bioinformatics. 2013:43(1):11-0.

44. Zerbino DR, Birney E. Velvet: algorithms for de novo short read assembly using de Bruijn graphs. Genome Res. 2008;18(5):821-9.

45. Li B, Dewey CN. RSEM: accurate transcript quantification from RNA-Seq data with or without a reference genome. BMC Bioinformatics. 2011;12(1):323.

46. Wang L, Feng Z, Wang X, Wang X, Zhang X. DEGseq: an R package for identifying differentially expressed genes from RNA-seq data. Bioinformatics. 2009:26(1):136-8.

47. Young MD, Wakefield MJ, Smyth GK, Oshlack A. Gene ontology analysis for RNA-seq: accounting for selection bias. Genome Biol. 2010;11(2):R14.

48. Mao X, Cai T, Olyarchuk JG, Wei L. Automated genome annotation and pathway identification using the KEGG Orthology (KO) as a controlled vocabulary. Bioinformatics. 2005;21(19):3787-93.
Ready to submit your research? Choose BMC and benefit from:
- fast, convenient online submission
- thorough peer review by experienced researchers in your field
- rapid publication on acceptance
- support for research data, including large and complex data types
- gold Open Access which fosters wider collaboration and increased citations
- maximum visibility for your research: over $100 \mathrm{M}$ website views per year
At BMC, research is always in progress. 